\title{
EXPLICIT CANONICAL METHODS FOR HAMILTONIAN SYSTEMS
}

\author{
DANIEL OKUNBOR AND ROBERT D. SKEEL
}

\begin{abstract}
We consider canonical partitioned Runge-Kutta methods for separable Hamiltonians $H=T(p)+V(q)$ and canonical Runge-Kutta-Nyström methods for Hamiltonians of the form $H=\frac{1}{2} p^{\mathrm{T}} M^{-1} p+V(q)$ with $M$ a diagonal matrix. We show that for explicit methods there is great simplification in their structure. Canonical methods of orders one through four are constructed. Numerical experiments indicate the suitability of canonical numerical schemes for long-time integrations.
\end{abstract}

\section{INTRODUCTION}

Time-independent Hamiltonian systems are of the form

$$
\frac{d}{d t} q_{i}=\frac{\partial H}{\partial p_{i}}(q, p), \quad \frac{d}{d t} p_{i}=-\frac{\partial H}{\partial q_{i}}(q, p),
$$

where $p, q \in \mathbf{R}^{N}$ and the Hamiltonian function $H$ is a continuously differentiable function of the generalized coordinates $q$ and the generalized momenta $p$. The $2 N$-dimensional space with coordinates $q_{1}, q_{2}, \ldots, q_{N}, p_{1}, p_{2}, \ldots$, $p_{N}$ is the phase space of the system. Let $z=\left(\begin{array}{l}q \\ p\end{array}\right)$. A transformation $z \rightarrow \bar{z}$ is said to be canonical if $\left(\frac{\partial \bar{z}}{\partial z}\right)^{\mathrm{T}} J\left(\frac{\partial \bar{z}}{\partial z}\right)=J$, where $J=\left(\begin{array}{cc}0 & I \\ -I & 0\end{array}\right)$.

The phase flow of (1),

$$
G_{t}:\left(\begin{array}{c}
q(0) \\
p(0)
\end{array}\right) \mapsto\left(\begin{array}{c}
q(t) \\
p(t)
\end{array}\right)
$$

is a one-parameter group of transformations of phase space. The transformation $G_{t}$ is canonical and by Liouville's theorem preserves volume in phase space, an important property of (1). The same is true of all canonical transformations. In terms of differential forms, the flow preserves the differential 2-form $\omega=$ $\sum d q^{i} \wedge d p^{i}$ (that is, the sum of areas of projections of any two-dimensional surface $S$ in phase space onto the $q_{i}-p_{i}$ planes), and all powers of $\omega$, whose integrals are the Poincare invariants $[1,6]$. In numerically solving (1), it may be important for the numerical integrators to be canonical so that, in particular,

Received by the editor February 21, 1991.

1991 Mathematics Subject Classification. Primary 65L05.

Key words and phrases. Hamiltonian systems, canonical methods, Runge-Kutta methods, adjoint methods, Runge-Kutta-Nyström methods.

This work was supported in part by National Science Foundation Grants DMS 8911410 and DMS 9015533. 
volume in phase space is preserved. This requirement was first considered by DeVogelaere (cf. [3]) and in a published paper by Ruth [11]. For information about the history of canonical numerical integrators, see [3] and its references.

A separable Hamiltonian has the form $H(q, p)=T(p)+V(q)$, where $T(p)$ and $V(q)$ are kinetic and potential energies, respectively. In this paper, we consider the case where $T(p)=\frac{1}{2} p^{\mathrm{T}} M^{-1} p$ with $M$ a diagonal matrix, and the resulting Hamiltonian system

$$
\dot{q}=M^{-1} p, \quad \dot{p}=-\frac{\partial V}{\partial q}
$$

is one describing the motion of $\frac{N}{3}$ particles. Examples of (2) abound in molecular dynamics, astronomy, etc.

The system (2) can be written as a second-order system thus:

$$
\ddot{q}=-M^{-1} \frac{\partial V}{\partial q}=f(q) .
$$

Suris [14] examined Runge-Kutta-Nyström (RKN) methods for (3) and obtained, using matrix algebra, the conditions for it to be canonical.

In our earlier paper [9], we obtained the same conditions using exterior forms. Furthermore, we showed that an explicit RKN method is canonical if and only if its adjoint is explicit. This paper builds on that work by using it to construct explicit schemes for (2). Section 2 gives a brief description of existing results for canonical Runge-Kutta-Nyström methods and shows that for explicit methods there is great simplification in their structure. Section 3 details the construction of methods of orders one through four. Section 4 reviews partitioned RungeKutta (pRK) methods for separable Hamiltonians proposed by Sanz-Serna [12] and shows again the simplification that occurs for explicit methods. Section 5 considers numerical illustrations.

\section{Canonical Runge-Kutta-Nyström Methods}

We associate with any given (one-step) method a mapping

$$
\Phi: z_{n}, h, H(p, q) \mapsto z_{n+1},
$$

where $h$ is the time step. This gives a transformation of $z$ from time $t_{n}$ to $t_{n+1}$. A method is said to be canonical if $\Phi=\Phi_{h}$ is a canonical mapping for any Hamiltonian $H$ and stepsize $h$. The method is of order $p$ if $\Phi_{h}$ differs from the phase flow $G_{h}$ by $O\left(h^{p+1}\right)$. It is becoming increasingly evident through numerical experiments $[3,12]$ that the dynamics of canonical $\Phi_{h}$ and $G_{h}$ are closely related in long-time integrations. For details of the advantages of canonical $\Phi_{h}$ over classical (noncanonical) methods, see [12]. In writing a method in the form (4), different methods may yield the same mapping $\Phi_{h}$. We shall identify a method with its $\Phi_{h}$ and the details of its internal stages. Since different methods may give the same mapping $\Phi_{h}$, it is essential to introduce the concepts of equivalence and redundancy. Two methods are said to be equivalent if their corresponding mappings $\Phi_{h}$ are identical. A method may be equivalent to a method of a lesser stage number. In this case, the method is regarded as redundant. 
An s-stage Runge-Kutta-Nyström method for (3) is given by

$$
y_{i}=q_{n}+c_{i} h \dot{q}_{n}+h^{2} \sum_{j=1}^{s} a_{i j} f\left(y_{j}\right), \quad i=1,2, \ldots, s,
$$

$$
\begin{aligned}
& q_{n+1}=q_{n}+h \dot{q}_{n}+h^{2} \sum_{i=1}^{s} b_{i} f\left(y_{i}\right), \\
& \dot{q}_{n+1}=\dot{q}_{n}+h \sum_{i=1}^{s} B_{i} f\left(y_{i}\right) .
\end{aligned}
$$

Naturally, we define $z_{n}=\left(\begin{array}{c}q_{n} \\ M \dot{q}_{n}\end{array}\right)$. The corresponding tableau $[2,8]$ is:

\begin{tabular}{c|cccc}
$c_{1}$ & $a_{11}$ & $a_{12}$ & $\cdots$ & $a_{1 s}$ \\
$c_{2}$ & $a_{21}$ & $a_{22}$ & $\cdots$ & $a_{2 s}$ \\
$\vdots$ & $\vdots$ & $\vdots$ & & $\vdots$ \\
$c_{s}$ & $a_{s 1}$ & $a_{s 2}$ & $\cdots$ & $a_{s s}$ \\
\hline & $b_{1}$ & $b_{2}$ & $\cdots$ & $b_{s}$ \\
& $B_{1}$ & $B_{2}$ & $\cdots$ & $B_{s}$
\end{tabular}

If we interchange $z_{n}$ and $h$ with $z_{n+1}$ and $-h$, respectively, in (4), we get the adjoint method [8]. Following are the coefficients of the adjoint method of (5):

$$
\begin{aligned}
\tilde{c}_{i} & =1-c_{s+1-i}, \\
\tilde{a}_{i j} & =B_{s+1-j}-b_{s+1-j}-c_{s+1-i} B_{s+1-j}+a_{s+1-i, s+1-j}, \\
\tilde{b}_{j} & =B_{s+1-j}-b_{s+1-j}, \quad i \leq i, j \leq s, \\
\widetilde{B}_{j} & =B_{s+1-j} .
\end{aligned}
$$

A method is time-reversible if $\tilde{c}_{i}=c_{i}, \tilde{a}_{i j}=a_{i j}, \tilde{b}_{i}=b_{i}$, and $\widetilde{B}_{i}=B_{i}$. We now give the conditions for an RKN method to be canonical, which are found in $[9,14]$.

Theorem 1 (Suris [14]). An s-stage RKN is canonical if and only if

$$
\begin{gathered}
b_{i}-B_{i}+c_{i} B_{i}=0, \quad 1 \leq i \leq s, \\
-B_{j} a_{j i}+B_{i} a_{i j}+B_{j} b_{i}-B_{i} b_{j}=0, \quad 1 \leq i<j \leq s .
\end{gathered}
$$

An RKN method is explicit if $a_{i j}=0$ for $j \geq i$. For an explicit RKN method, equation $(7 \mathrm{~b})$ reduces to

$$
B_{i} a_{i j}+B_{j} b_{i}-B_{i} b_{j}=0, \quad j<i .
$$


From equations (7a) and (8), we get

$$
\begin{gathered}
b_{i}=B_{i}\left(1-c_{i}\right), \\
B_{i} a_{i j}=B_{i} B_{j}\left(c_{i}-c_{j}\right), \quad j<i .
\end{gathered}
$$

We would like to conclude from the second of these two that $a_{i j}=B_{j}\left(c_{i}-c_{j}\right)$, but that would not be quite correct. What can be proved is that the method is equivalent to one with this choice for $a_{i j}$.

Lemma 1. An explicit canonical $(r+1)$-stage $R K N$ method (5) is equivalent to

$$
\begin{aligned}
y_{i} & =q_{n}+c_{i} h \dot{q}_{n}+h^{2} \sum_{j=1}^{i-1} a_{i j} f\left(y_{j}\right), \quad i=1,2, \ldots, r, \\
y_{r+1} & =q_{n}+c_{r+1} h \dot{q}_{n}+h^{2} \sum_{j=1}^{r} B_{j}\left(c_{r+1}-c_{j}\right) f\left(y_{j}\right), \\
q_{n+1} & =q_{n}+h \dot{q}_{n}+h^{2} \sum_{i=1}^{r+1} B_{i}\left(1-c_{i}\right) f\left(y_{i}\right), \\
\dot{q}_{n+1} & =\dot{q}_{n}+h \sum_{i=1}^{r+1} B_{i} f\left(y_{i}\right) .
\end{aligned}
$$

Proof. It is enough to show that

$$
\begin{array}{r}
B_{r+1} f\left(q_{n}+c_{r+1} h \dot{q}_{n}+h^{2} \sum_{j=1}^{r} B_{j}\left(c_{r+1}-c_{j}\right) f\left(y_{j}\right)\right) \\
=B_{r+1} f\left(q_{n}+c_{r+1} h \dot{q}_{n}+h^{2} \sum_{j=1}^{r} a_{r+1, j} f\left(y_{j}\right)\right) .
\end{array}
$$

From (9b),

$$
\text { either } \quad B_{r+1}=0 \quad \text { or } \quad a_{r+1, j}=B_{j}\left(c_{r+1}-c_{j}\right), \quad j<r+1,
$$

and the result follows.

Theorem 2. An explicit canonical $R K N$ method is equivalent to

$$
\begin{aligned}
y_{i} & =q_{n}+c_{i} h \dot{q}_{n}+h^{2} \sum_{j=1}^{i-1} B_{j}\left(c_{i}-c_{j}\right) f\left(y_{j}\right), \quad i=1,2, \ldots, s, \\
q_{n+1} & =q_{n}+h \dot{q}_{n}+h^{2} \sum_{i=1}^{s} B_{i}\left(1-c_{i}\right) f\left(y_{i}\right), \\
\dot{q}_{n+1} & =\dot{q}_{n}+h \sum_{i=1}^{s} B_{i} f\left(y_{i}\right) .
\end{aligned}
$$

Proof. Repeatedly apply Lemma 1 and use the fact that each embedded method, namely 


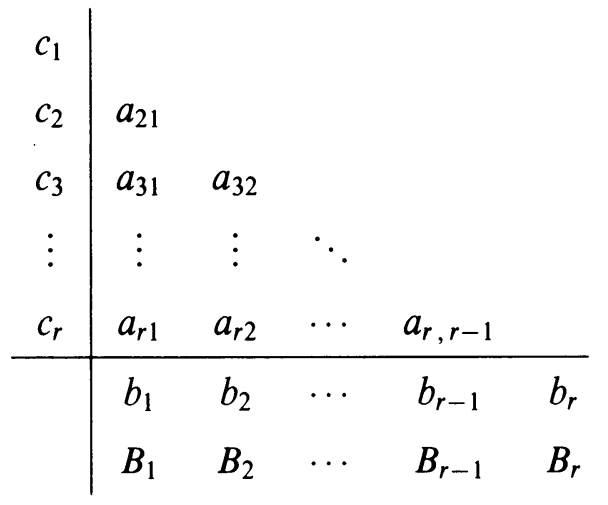

is itself canonical.

The only free parameters for a canonical explicit RKN method are therefore $B_{i}$ and $c_{i}$. The form (11) of writing an explicit canonical RKN method enables us to prove the following propositions. The first proposition shows that a canonical explicit RKN method requires minimal possible storage of any method.

Proposition 1. The explicit canonical RKN method (11) can be expressed as

$$
\begin{aligned}
& y_{0}=q_{n}, \\
& \dot{y}_{n}=\dot{q}_{n}, \\
& \text { for } i=1,2, \ldots, s \\
& \quad y_{i}=y_{i-1}+h\left(c_{i}-c_{i-1}\right) \dot{y}_{i-1}, \quad \text { where } c_{0}=0, \\
& \quad \dot{y}_{i}=\dot{y}_{i-1}+h B_{i} f\left(y_{i}\right), \\
& q_{n+1}=y_{s}+h\left(1-c_{s}\right) \dot{y}_{s}, \\
& \dot{q}_{n+1}=\dot{y}_{s} .
\end{aligned}
$$

Proof. We can always write down the above method. We need to show that it computes the same $\left(q_{n+1}, \dot{q}_{n+1}\right)$ as (5):

$$
\begin{aligned}
\dot{y}_{i} & =\dot{q}_{n}+h \sum_{j=1}^{i} B_{j} f\left(y_{j}\right) \\
y_{i} & =q_{n}+h \sum_{k=1}^{i}\left(c_{k}-c_{k-1}\right) \dot{y}_{k-1} \\
& =q_{n}+h\left(c_{i}-c_{0}\right) \dot{q}_{n}+h^{2} \sum_{k=1}^{i}\left(c_{k}-c_{k-1}\right) \sum_{j=1}^{k-1} B_{j} f\left(y_{j}\right) \\
& =q_{n}+h c_{i} \dot{q}_{n}+h^{2} \sum_{j=1}^{i-1}\left(c_{i}-c_{j}\right) B_{j} f\left(y_{j}\right) \\
& =q_{n}+h c_{i} \dot{q}_{n}+h^{2} \sum_{j=1}^{i-1} a_{i j} f\left(y_{j}\right)
\end{aligned}
$$




$$
\begin{aligned}
\dot{q}_{n+1}= & \dot{q}_{n}+h \sum_{j=1}^{s} B_{j} f\left(y_{j}\right) \\
q_{n+1}= & q_{n}+h c_{s} \dot{q}_{n}+h^{2} \sum_{j=1}^{s-1} a_{s j} f\left(y_{j}\right)+h\left(1-c_{s}\right) \\
& \times\left(\dot{q}_{n}+h \sum_{j=1}^{i} B_{j} f\left(y_{j}\right)\right) \\
= & q_{n}+h \dot{q}_{n}+h^{2} \sum_{j=1}^{s}\left(B_{j} c_{s}-B_{j} c_{j}+\left(1-c_{s}\right) B_{j}\right) \\
= & q_{n}+h \dot{q}_{n}+h^{2} \sum_{j=1}^{s} b_{j} f\left(y_{j}\right) .
\end{aligned}
$$

From Proposition 1, if either $c_{i}=c_{i-1}$ or $B_{i}=0$, then the $i$ th stage is redundant, and hence the following assertion.

Proposition 2. A nonredundant explicit canonical $R K N$ method has $B_{i} \neq 0$, $i=1,2, \ldots, s$, and $c_{i} \neq c_{i-1}, i=2,3, \ldots, s$.

Suris, in his paper [13], showed that an explicit RKN method is Liouville (i.e., volume preserving) if and only if its adjoint is equivalent to an explicit method. Canonical RKN methods form a subset of Liouville RKN methods. For example, explicit 2-stage RKN methods require $c_{1}=c_{2}$ or $B_{2} a_{21}+B_{2} b_{1}-$ $B_{1} b_{2}$ to be Liouville. What this means is that if we choose $c_{1}$ so that it is equal to $c_{2}$, then the method is Liouville but may or may not be canonical. The method

\begin{tabular}{l|ll}
$\frac{1}{2}$ & 0 & \\
$\frac{1}{2}$ & $\frac{1}{2}$ & 0 \\
\hline & $\frac{1}{4}$ & $\frac{1}{4}$ \\
& $\frac{1}{2}$ & $\frac{1}{2}$
\end{tabular}

is Liouville but not canonical. The following results are found in [9].

Theorem 3. If a method is canonical, then its adjoint is canonical.

Theorem 4. An explicit RKN method is canonical if and only if its adjoint is explicit.

A corollary of Theorem 4 is

Corollary 1. If an explicit $R K N$ method is equal to its adjoint (symmetric), then it is canonical.

Because the adjoint of an explicit canonical RKN method is explicit, it can be expressed entirely in terms of its coefficients $\widetilde{B}_{i}$ and $\tilde{c}_{i}$. These are given by (6) as

$$
\tilde{c}_{i}=1-c_{s+1-i}, \quad \widetilde{B}_{j}=B_{s+1-j}
$$




\section{CONSTRUCTION OF CANONICAL RKN METHOdS}

Order conditions for RKN methods are specified in [8] for $q$ and $\dot{q}$ involving $b_{i}$ and $B_{i}$. To construct explicit canonical RKN methods, we need only satisfy the conditions involving $B_{i}$. This is because $b_{i}=B_{i}\left(1-c_{i}\right)$ for canonical methods, and according to [8, p. 268] the other order conditions, involving $b_{i}$, are automatically satisfied. For an $s$-stage canonical RKN method we are left with the following conditions. The first-order condition is

$$
\sum B_{i}=1 \text {. }
$$

In addition to the first-order condition, the second-order conditions are

$$
\sum B_{i} c_{i}=\frac{1}{2} \text {. }
$$

The third-order conditions, in addition to the first- and second-order conditions, are

$$
\begin{gathered}
\sum B_{i} c_{i}^{2}=\frac{1}{3}, \\
\sum \sum B_{i} a_{i j}=\sum \sum_{j<i} B_{i} B_{j}\left(c_{i}-c_{i}\right)=\frac{1}{6} .
\end{gathered}
$$

Finally, the fourth-order conditions, in addition to first-, second-, and thirdorder conditions, are

$$
\begin{gathered}
\sum B_{i} c_{i}^{3}=\frac{1}{4}, \\
\sum \sum B_{i} c_{i} a_{i j}=\sum \sum_{j<i} B_{i} B_{j} c_{i}\left(c_{i}-c_{j}\right)=\frac{1}{8}, \\
\sum \sum B_{i} a_{i j} c_{j}=\sum \sum_{j<i} B_{i} B_{j}\left(c_{i}-c_{j}\right) c_{j}=\frac{1}{24} .
\end{gathered}
$$

The last of these turns out to be superfluous because we have

$$
\begin{aligned}
\sum \sum_{j<i} B_{i} B_{j}\left(c_{i}-c_{j}\right) c_{j} & =-\sum \sum_{j<i} B_{i} B_{j} c_{i}\left(c_{i}-c_{j}\right) \\
& =\sum \sum_{j<i} B_{i} B_{j} c_{i}\left(c_{i}-c_{j}\right)-\sum \sum B_{i} B_{j} c_{i}\left(c_{i}-c_{j}\right) \\
& =\frac{1}{8}-\left(\frac{1}{3} \cdot 1-\frac{1}{2} \cdot \frac{1}{2}\right)=\frac{1}{24},
\end{aligned}
$$

if all the others are satisfied.

There is no limit to the order attainable by a canonical explicit RKN method. By means of a Lie group analysis, Yoshida [15] gives a construction of a $3^{n}$-stage method of order $2 n+2$ for any $n$. See also Forest and Ruth [4].

3.1. One-stage methods. There is a one-parameter family of canonical explicit 1-stage RKN methods of order 1: $c_{1}$ is the free parameter, $B_{1}=1$. Imposing the second-order condition gives a canonical 1-stage RKN method of order 2 with $c_{1}=\frac{1}{2}$. This is equivalent to the Störmer, "leapfrog," or Verlet method commonly used for solving Newton's equations of motion occurring in molecular dynamics. 
3.2. Two-stage methods. We have a two-parameter family of canonical explicit 2-stage RKN methods of order 2, namely,

$$
c_{1}=\frac{1}{2}+\alpha, \quad c_{2}=\frac{1}{2}+\beta, \quad B_{1}=\frac{\beta}{\beta-\alpha}, \quad B_{2}=\frac{-\alpha}{\beta-\alpha}, \quad \alpha \neq \beta .
$$

A simple choice for $\alpha$ and $\beta$ results in the method

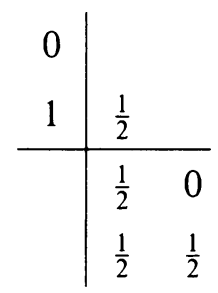

This is another form of the Störmer-Verlet method. The only explicit 2-stage RKN of order 3 that is canonical has imaginary coefficients, which are difficult to work with.

3.3. Three-stage methods. Imposing third-order conditions, except for (16b) and canonical conditions, we obtain a three-parameter family of explicit 3-stage Runge-Kutta-Nyström methods with the coefficients

$$
\begin{gathered}
c_{1}=\frac{1}{2}+\alpha, \quad c_{2}=\frac{1}{2}+\beta, \quad c_{3}=\frac{1}{2}+\gamma, \\
B_{1}=\frac{\frac{1}{2}+\beta \gamma}{(\beta-\alpha)(\gamma-\alpha)}, \quad B_{2}=-\frac{\frac{1}{12}+\alpha \gamma}{(\beta-\alpha)(\gamma-\beta)}, \quad B_{3}=\frac{\frac{1}{12}+\alpha \beta}{(\gamma-\alpha)(\gamma-\beta)},
\end{gathered}
$$

where $\alpha, \beta$, and $\gamma$ are all distinct. Through (16b), the parameters $\alpha, \beta$, and $\gamma$ are constrained by the following nonlinear equation:

$$
1+24 \alpha \gamma+24(\beta-\alpha)(\gamma-\alpha)(\gamma-\beta)+144 \alpha \beta \gamma(\alpha+\gamma-\beta)=0 .
$$

If $\gamma=\alpha$, that is, $c_{1}=c_{3}$, then from equations (14) and (15) we get

$$
B_{1}+B_{3}=\frac{c_{2}-\frac{1}{2}}{c_{2}-c_{1}}, \quad B_{2}=\frac{\frac{1}{2}-c_{1}}{c_{2}-c_{1}} .
$$

With this, the order condition $(16 \mathrm{~b})$ reduces to

$$
\left(\frac{1}{2}-c_{1}\right)\left(B_{1}-B_{3}\right)=\frac{1}{6} \text {. }
$$

Combining (18) and (19), we solve for the coefficients to obtain a two-parameter family of 3-stage RKN methods. The coefficients are

$$
\begin{gathered}
c_{1}=\frac{1}{2}+\alpha, \quad c_{2}=\frac{1}{2}+\beta, \quad c_{3}=\frac{1}{2}+\alpha, \\
B_{1}=\frac{\beta}{2(\beta-\alpha)}-\frac{1}{12 \alpha}, \quad B_{2}=-\frac{\alpha}{(\beta-\alpha)}, \quad B_{3}=\frac{\beta}{2(\beta-\alpha)}+\frac{1}{12 \alpha},
\end{gathered}
$$

where $\alpha \neq \beta$ and $\alpha \neq 0$. Condition (16a) constrains the parameters $\alpha$ and $\beta$ by $\frac{1}{12}+\alpha \beta=0$ and makes the method of order 3 . 
For a symmetric canonical explicit 3-stage RKN method (that is, an RKN method which is equal to its adjoint), it is sufficient to impose in addition to (17) only two conditions, namely, $\alpha=-\gamma$ and $\beta=0$. Solving for the coefficients, we have

$$
\begin{gathered}
c_{1}=\frac{1}{2}-\gamma, \quad c_{2}=\frac{1}{2}, \quad c_{3}=\frac{1}{2}+\gamma, \\
B_{1}=\frac{1}{24 \gamma^{2}}, \quad B_{2}=1-\frac{1}{12 \gamma^{2}}, \quad B_{3}=\frac{1}{24 \gamma^{2}},
\end{gathered}
$$

where $\gamma$ is a zero of $p(x)=48 x^{3}-24 x^{2}+1$. The only real zero of this polynomial is $\frac{1}{12}(2-\sqrt[3]{4}-\sqrt[3]{16}) \approx-0.1756035959798288$. It is easily verified from (16a), (16b), and (16c) that this is a symmetric canonical 3-stage RKN method of order four (we use the acronym SYRKN for this method). This 3stage fourth-order method was apparently [15] first discovered by E. Forest and first published by Forest and Ruth [4].

\section{Partitioned RK methods}

Consider a separable Hamiltonian system

$$
\dot{q}=\frac{\partial T}{\partial p}=G(p), \quad \dot{p}=-\frac{\partial V}{\partial q}=F(q) .
$$

In what follows, we give an overview of partitioned Runge-Kutta (pRK) methods for (20), proposed by Sanz-Serna [12], an approach which is actually a generalization of a 3-stage RK method obtained by Ruth [11] using generating functions via Hamilton-Jacobi equations. Sanz-Serna [12] considered 3-stage, and Qin and Zhang [10] considered 4-stage, pRK methods of order four. Here, we provide results concerning this class of methods.

Definition 1 (Sanz-Serna [12]). An $s$-stage partitioned RK method is

$$
\begin{array}{rlrl}
Y_{i} & =q_{n}+h \sum_{j} a_{i j} G\left(Z_{j}\right), & Z_{i} & =p_{n}+h \sum_{j} A_{i j} F\left(Y_{j}\right), \\
q_{n+1} & =q_{n}+h \sum_{i} b_{i} G\left(Z_{i}\right), \quad p_{n+1} & =p_{n}+h \sum_{i} B_{i} F\left(Y_{i}\right) .
\end{array}
$$

Note that this formalism includes those pRK methods that use an unequal number of $G$ and $F$ evaluations to take one step; it is only necessary to insert appropriate dummy stages. A pRK method is said to be explicit if $a_{i j}=0$ for $j>i$ and $A_{i j}=0$ for $j \geq i$. It is true that there are methods equivalent to explicit methods which do not satisfy this. However, for simplicity we shall restrict the term "explicit" to methods of this form. The Butcher array for a partitioned RK method is

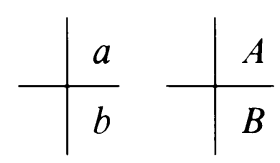

Sanz-Serna in his paper [12] showed that method (21) is canonical if

$$
B_{j} a_{j i}+b_{i} A_{i j}-B_{j} b_{i}=0, \quad 1 \leq i, j \leq s .
$$


As pointed out in [12], if we define $\bar{q}(\bar{t})=p(-\bar{t})$ and $\bar{p}(\bar{t})=q(-\bar{t})$, these satisfy a Hamiltonian system with Hamiltonian $\bar{H}(\bar{q}, \bar{p})=H(\bar{p}, \bar{q})$. If we apply a one-step method to this transformed system, we get equations for $\bar{q}_{n+1}$ and $\bar{p}_{n+1}$ in terms of $\bar{q}_{n}$ and $\bar{p}_{n}$. For these numerically determined values we could then undo the transformation by the substitutions $\bar{q}_{n+1} \rightarrow p_{n}, \bar{p}_{n+1} \rightarrow q_{n}$, $\bar{q}_{n} \rightarrow p_{n+1}, \bar{p}_{n} \rightarrow q_{n+1}$, and $\bar{H}(\bar{q}, \bar{p}) \rightarrow H(\bar{p}, \bar{q})$. It turns out that $q_{n+1}$ and $p_{n+1}$ are given in terms of $q_{n}$ and $p_{n}$ by a pRK method with coefficients

$$
\begin{array}{cc}
\tilde{a}_{i j}=B_{s+1-j}-A_{s+1-i, s+1-j}, & \tilde{b}_{j}=B_{s+1-j}, \\
\widetilde{A}_{i j}=b_{s+1-j}-a_{s+1-i, s+1-j}, & \widetilde{B}_{j}=b_{s+1-j} .
\end{array}
$$

We call this the $G$-adjoint method. A $G$-symmetric method is one which is equal to its G-adjoint. It satisfies conditions which reduce to

$$
\begin{aligned}
& a_{i j}=b_{j}-A_{s+1-i, s+1-j}, \quad 1 \leq i, j \leq s . \\
& A_{i j}=B_{j}-a_{s+1-i, s+1-j},
\end{aligned}
$$

The justification for a G-symmetric method is that if a problem has two equivalent formulations, then it seems desirable that a method applied to both formulations should yield equivalent solutions.

If we impose canonical conditions (22) on an explicit pRK method, we have

$$
B_{i} a_{i j}=B_{i} b_{j} \quad \text { for } j \leq i, \quad b_{i} A_{i j}=b_{i} B_{j} \quad \text { for } j<i, \quad 1 \leq i \leq s .
$$

As with RKN methods, we cannot conclude that $a_{i j}=b_{j}$, nor that $A_{i j}=B_{j}$, but we can prove that the method is equivalent to one with this choice of coefficients. Such a choice is mentioned in [12].

Lemma 2. Let

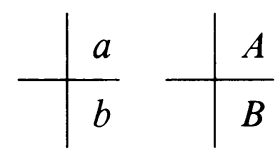

be an explicit canonical $(r+1)$-stage pRK method. This is equivalent to

\begin{tabular}{c|ccc|c}
$a_{11}$ & $\cdots$ & $a_{1 r}$ & 0 \\
$\vdots$ & & $\vdots$ & $\vdots$ \\
& $a_{r 1}$ & $\cdots$ & $a_{r r}$ & 0 \\
\hline & $b_{1}$ & $\cdots$ & $b_{r}$ & $b_{r+1}$ \\
\hline & $b_{1}$ & $\cdots$ & $b_{r}$ & $b_{r+1}$
\end{tabular}

\begin{tabular}{c|ccc|c} 
& $A_{11}$ & $\cdots$ & $A_{1 r}$ & 0 \\
$\vdots$ & & $\vdots$ & $\vdots$ \\
& $A_{r 1}$ & $\cdots$ & $A_{r r}$ & 0 \\
\hline & $B_{1}$ & $\cdots$ & $B_{r}$ & 0 \\
\hline & $B_{1}$ & $\cdots$ & $B_{r}$ & $B_{r+1}$
\end{tabular}

Proof. It is enough to show that

$$
b_{r+1} G\left(p_{n}+h \sum_{j=1}^{r} A_{r+1, j} F_{j}\right)=b_{r+1} G\left(p_{n}+h \sum_{j=1}^{r} B_{j} F_{j}\right),
$$


and

$$
B_{r+1} F\left(q_{n}+h \sum_{j=1}^{r+1} a_{r+1, j} G_{j}\right)=B_{r+1} F\left(q_{n}+h \sum_{j=1}^{r+1} b_{j} G_{j}\right) .
$$

From (23) we know that

$$
\text { either } b_{r+1}=0 \quad \text { or } \quad A_{r+1, j}=B_{j}, \quad j<r+1 \text {, }
$$

and

$$
\text { either } \quad B_{r+1}=0 \quad \text { or } \quad a_{r+1, j}=b_{j}, \quad j \leq r+1 .
$$

Theorem 5. An explicit canonical pRK method

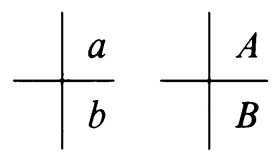

is equivalent to

$$
\mid \begin{array}{ccccc}
b_{1} & & & & \\
b_{1} & b_{2} & & & \\
b_{1} & b_{2} & b_{3} & & \\
\vdots & \vdots & \ddots & \ddots & \\
b_{1} & b_{2} & \cdots & b_{s-1} & b_{s} \\
\hline b_{1} & b_{2} & \cdots & b_{s-1} & b_{s}
\end{array}
$$

$$
\mid \begin{array}{ccccc}
0 & & & & \\
B_{1} & 0 & & & \\
B_{1} & B_{2} & 0 & & \\
\vdots & \vdots & \ddots & \ddots & \\
B_{1} & B_{2} & \cdots & B_{s-1} & 0 \\
\hline B_{1} & B_{2} & \cdots & B_{s-1} & B_{s}
\end{array}
$$

Proof. Repeatedly apply Lemma 2.

Without loss of generality, the algorithm for an explicit canonical pRK method can be written as

$$
\begin{aligned}
& \quad \begin{array}{l}
Y_{0}=q_{n}, \\
Z_{1}=p_{n}, \\
\text { for } i=1,2, \ldots, s \\
\quad Y_{i}=Y_{i-1}+h b_{i} G\left(Z_{i}\right), \\
\quad Z_{i+1}=Z_{i}+h B_{i} F\left(Y_{i}\right), \\
q_{n+1}=Y_{s}, \\
p_{n+1}=Z_{s+1} .
\end{array}
\end{aligned}
$$

Note that this algorithm requires minimal possible storage.

There is an obvious correspondence between this and the algorithm of Proposition 1. Every explicit canonical $r$-stage RKN method comes from an explicit 
canonical $(r+1)$-stage pRK method with $B_{i}$ the same for $i=1,2, \ldots, r$ and $B_{r+1}=0$, and with $b_{i}=c_{i}-c_{i-1}$ for $i=1,2, \ldots, r, r+1$, where $c_{0}=0$ and $c_{r+1}=1$. (If $c_{r}=1$, then the $(r+1)$ st stage is not needed.) We can characterize either an explicit RKN or an explicit pRK method in terms of parameters

$$
\begin{array}{lllll}
b: & b_{1} & b_{2} & \cdots & b_{s} \\
B: & B_{1} & B_{2} & \cdots & B_{s}
\end{array}
$$

rather than the Butcher tableau. The G-adjoint of the explicit pRK method above has parameters

$$
\begin{array}{lllll}
\tilde{b}: & B_{s} & B_{s-1} & \cdots & B_{1} \\
\widetilde{B}: & b_{s} & b_{s-1} & \cdots & b_{1}
\end{array} .
$$

The adjoint has parameters

$$
\begin{array}{cccccc}
\tilde{b}: & 0 & b_{s} & b_{s-1} & \cdots & b_{1} \\
\widetilde{B}: & B_{s} & B_{s-1} & \cdots & B_{1} & 0 .
\end{array}
$$

For the special case $T(p)=\frac{1}{2} p^{\mathrm{T}} M^{-1} p$ we have available the explicit RKN order conditions in terms of $c_{i}$ and $B_{i}$, where $c_{i}=\sum_{j=1}^{i} b_{i}$ if we assume that $\sum_{j=1}^{s} b_{j}=1$ (which is needed for consistency).

Sanz-Serna [12] has suggested a concatenation of a $G$-symmetric 3-stage pRK method of order 3 ,

$$
\begin{array}{llll}
b: & 0.268330 & -0.187992 & 0.919662 \\
B: & 0.919662 & -0.187992 & 0.268330,
\end{array}
$$

with its adjoint method to get a method of order 4 (we give it the acronym SYPRK1):

$$
\begin{array}{lrrrrrr}
b: & 0.134165 & -0.093996 & 0.459831 & 0.459831 & -0.093996 & 0.134165 \\
B: & 0.459831 & -0.093996 & 0.268330 & -0.093996 & 0.459831 & 0 .
\end{array}
$$

(These coefficients are approximate.) We suggest another method which is a concatenation of a method obtained by Ruth [11], namely,

$$
\begin{array}{cccc}
b: & \frac{7}{24} & \frac{3}{4} & -\frac{1}{24} \\
B: & \frac{2}{3} & -\frac{2}{3} & 1,
\end{array}
$$

and its adjoint. The result of this concatenation is a method of order 4 (acronym: SYPRK2):

$$
\begin{array}{ccccccc}
b: & \frac{7}{48} & \frac{3}{8} & -\frac{1}{48} & -\frac{1}{48} & \frac{3}{8} & \frac{7}{48} \\
B: & \frac{1}{3} & -\frac{1}{3} & 1 & -\frac{1}{3} & \frac{1}{3} & 0
\end{array}
$$

\section{NUMERICAL EXPERIMENTS}

For illustrative purposes, we consider two well-known Hamiltonians, the Hénon-Heiles and a Kepler Hamiltonian. The experiments considered are not exhaustive and do not erase all doubts about the advantages of canonical over noncanonical schemes. More work needs to be done. For these experiments we consider also a noncanonical RKN of order four (acronym: NCRKN) obtained from [8, p. 262]. 
5.1. Hénon-Heiles Hamiltonian. In the process of investigating the existence of a third isolating integral of galactic motion in celestial mechanics, Henon and Heiles [7] approximated the Hamiltonian, and hence the total energy, by

$$
H=\frac{1}{2}\left(p_{1}^{2}+p_{2}^{2}\right)+\frac{1}{2}\left(q_{1}^{2}+q_{2}^{2}+2 q_{1}^{2} q_{2}-\frac{2}{3} q_{2}^{3}\right) .
$$

Hénon and Heiles found that a third integral exists only at low energy. For energies higher than $\frac{1}{8}$, the system exhibits a chaotic behavior. The reason for considering it here is numerical and has nothing to do with theoretical questions. Hénon and Heiles [7] in their experiments considered the intersections of the trajectory with the plane $q_{1}=0$. They plotted the values of $q_{2}$ and $p_{2}$ at these intersections in the $\left(q_{2}, p_{2}\right)$-plane. We shall do likewise, in addition to the quantities computed by Sanz-Serna [12].

Similar to [3], we choose $\left(q_{1}, q_{2}, p_{1}, p_{2}\right)=(0.12,0.12,0.12,0.12)$, giving an energy of 0.029952 . The solution is computed for $1,200,000$ time steps, with the time step being $\frac{1}{6}$. Figure 1 depicts the Poincare sections of the
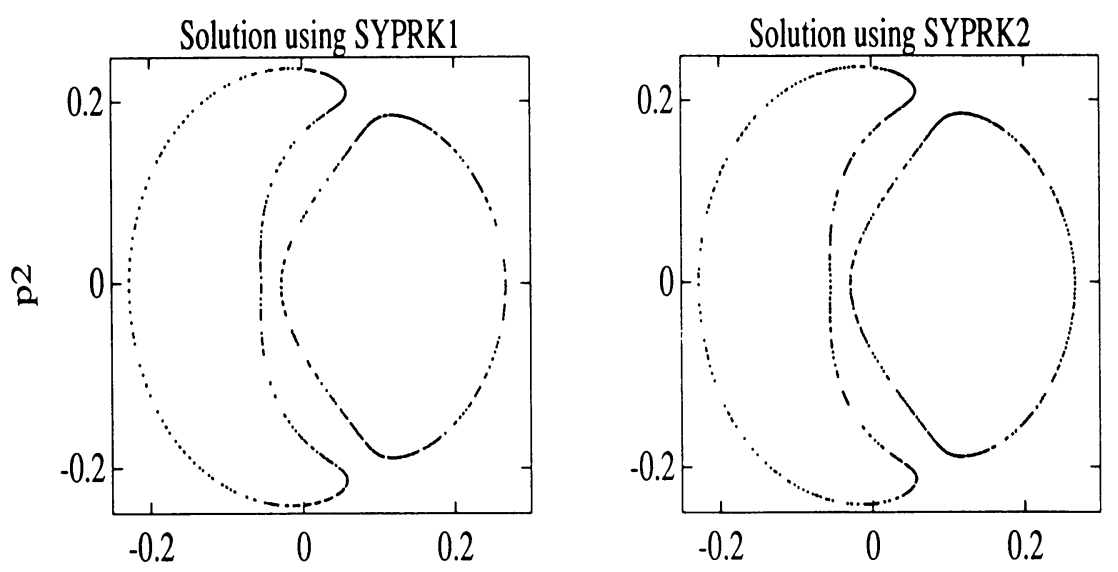

q2
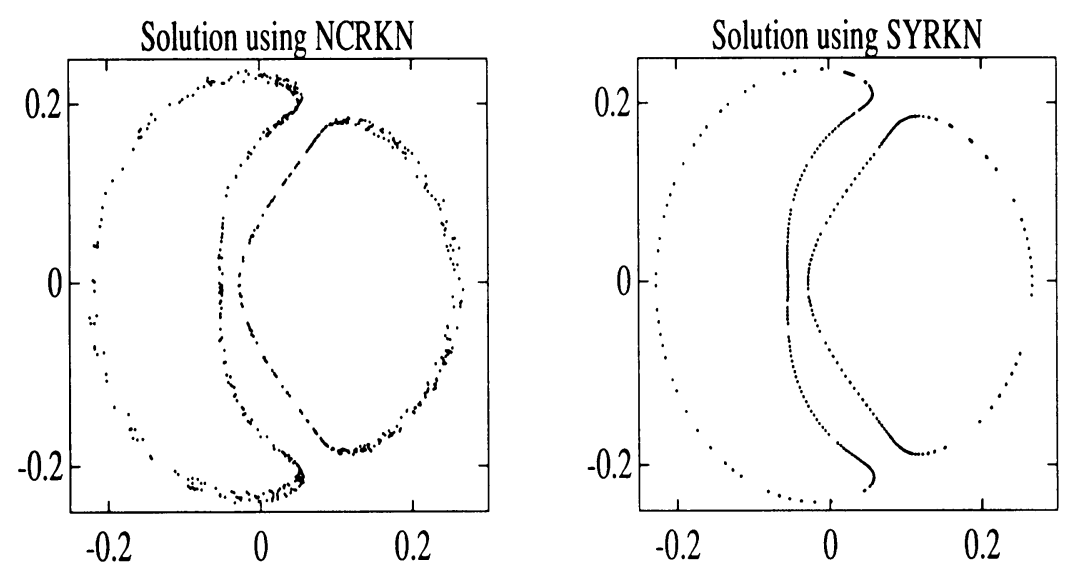

FIGURE 1. Two-dimensional surface plot for the HénonHeiles problem using canonical and noncanonical methods $(H=0.029952)$ 

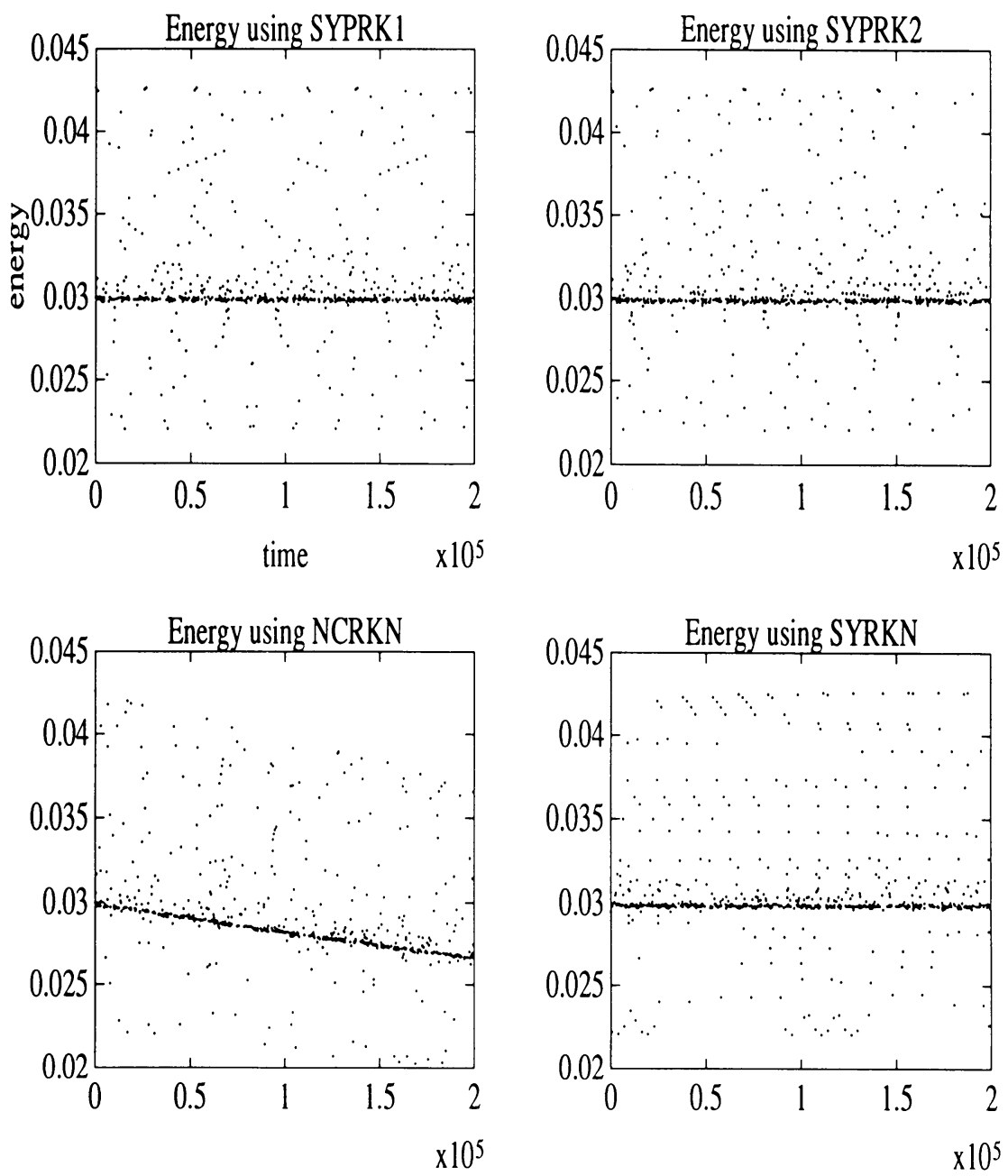

Figure 2. Energy plots for the Hénon-Heiles problem using canonical and noncanonical methods $(H=$ 0.029952 )

$\left(q_{2}, p_{2}\right)$-plane, using SYPRK1, SYPRK2, SYRKN, and NCRKN. As expected, the noncanonical scheme gives solution points which lie on the curves for some time and gradually drift away from the two submanifolds, displaying, perhaps, the noncanonical effects, a phenomenon common with such methods [11]. On the other hand, the solution points computed by all the canonical schemes lie on the two submanifolds. It is hoped that this feature would remain so for an infinite number of time steps. Figure 2 represents the energy behavior of all the schemes. This clearly shows the significance of canonical schemes for long-time computations.

We also solve the problem choosing

$$
\left(q_{1}, q_{2}, p_{1}, p_{2}\right)=(0,0.2,0.4483395,0),
$$

where $p_{1}$ was computed so that $H=0.117835$. The number of time steps is 5,000 with $h=0.5$. Figure 3 shows the trajectories on the $\left(q_{1}, q_{2}\right)$-plane using all the methods. 

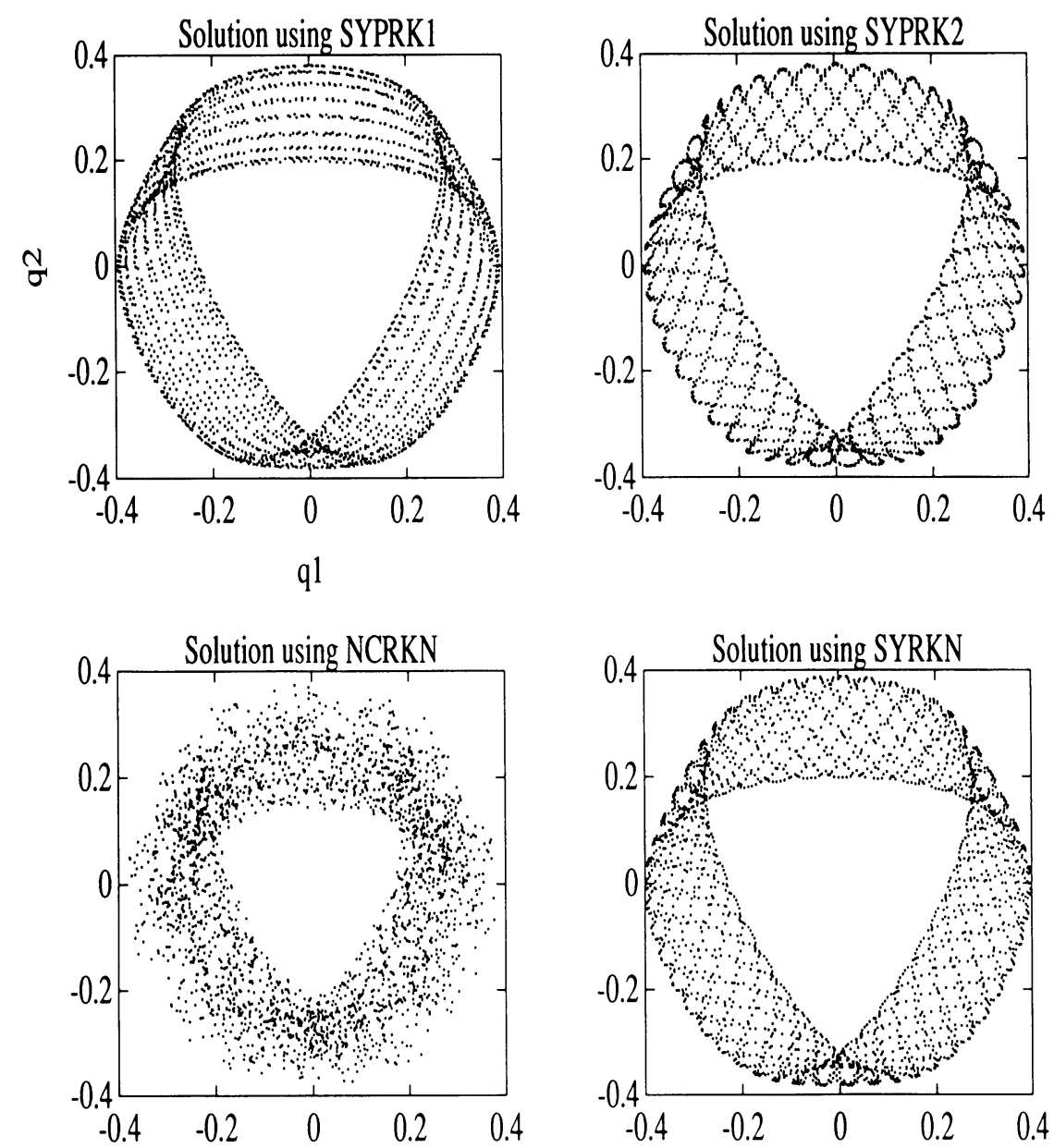

Figure 3. Trajectories for the Hénon-Heiles problem using canonical and noncanonical methods $(H=0.117835)$

5.3. Kepler's problem. The motion of two bodies under mutual gravitational attraction is governed by the Hamiltonian

$$
H=\frac{1}{2}\left(p_{1}^{2}+p_{2}^{2}\right)-\frac{\alpha}{\left(q_{1}^{2}+q_{2}^{2}\right)^{1 / 2}},
$$

where $\alpha$ is a constant involving the gravitational constant [5, pp. 132, 133], the masses of the bodies, and units of measurement. Different choices of initial conditions lead to different solution orbits. For this problem, we use $\left(q_{1}, q_{2}, p_{1}, p_{2}\right)=\left(0.75,0,0, \alpha \sqrt{\frac{5}{3}}\right)$ as initial conditions and $\frac{\pi}{4}$ for $\alpha$. The solution orbit is an ellipse with low eccentricity of 0.25 and a focus at the origin. The solution is periodic with period $T=8$. The plots of the global error (i.e., $\left\|\mathbf{q}\left(t_{n}\right)-\mathbf{q}_{n}\right\| /\left\|\mathbf{q}\left(t_{n}\right)\right\|$ ) versus time, using SYPRK1, SYPRK2, NCRKN, and SYRKN with scaled stepsize (i.e., the actual stepsize divided by the number of stages of a method) of $\frac{T}{450}$ are shown in Figure 4 (see next page). The solution was computed for 100 time periods. It is remarkable to see from the 


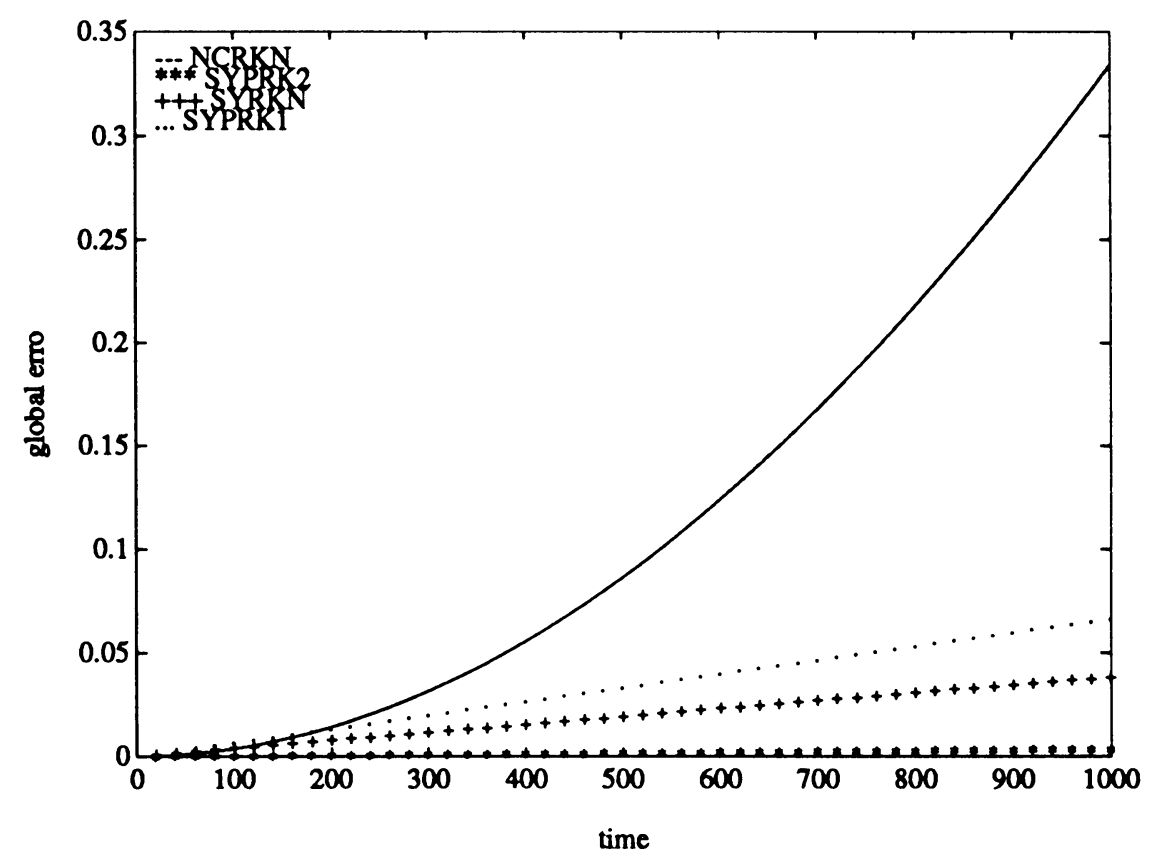

FIGURE 4. Error analysis of canonical and noncanonical schemes (Kepler's problem)

figure that, while canonical methods exhibit linear growth in global error, the noncanonical method gave an exponential growth. This again shows the advantage of canonical numerical mappings over noncanonical ones for long-time computation.

\section{BIBLIOGRAPHY}

1. V. I. Arnold, Mathematical methods of classical mechanics, Springer-Verlag, Berlin, 1989.

2. J. C. Butcher, The numerical analysis of ordinary differential equations: Runge-Kutta and general linear methods, Wiley, New York, 1987.

3. P. J. Channell and J. C. Scovel, Symplectic integration of Hamiltonian systems, Nonlinearity 3 (1990), 231-259.

4. E. Forest and R. D. Ruth, Fourth order symplectic integration, Phys. D 43 (1990), 105-117.

5. G. E. Forsythe, M. A. Malcolm, and C. B. Moler, Computer methods for mathematical computations, Prentice-Hall, Englewood Cliffs, NJ, 1977.

6. H. Goldstein, Classical mechanics, Addison-Wesley, Reading, MA, 1965.

7. M. Hénon and C. Heiles, The applicability of third integral of motion: some numerical experiments, Astronom. J. 69 (1964), 73-79.

8. E. Hairer, S. P. Nørsett, and G. Wanner, Solving ordinary differential equations I: Non-stiff systems, Springer-Verlag, Berlin, 1987.

9. D. Okunbor and R. D. Skeel, An explicit Runge-Kutta-Nyström method is canonical if and only if its adjoint is explicit, SIAM J. Numer. Anal. 29 (1992), 521-527.

10. M.-Z. Qin and M.-Q. Zhang, Symplectic Runge-Kutta schemes for Hamiltonian systems, preprint, 1989.

11. R. D. Ruth, A canonical integration technique, IEEE Trans. Nuclear Sci. 30 (1983), 26692671. 
12. J. M. Sanz-Serna, The numerical integration of Hamiltonian systems, Proc. IMA Conf. on Computational ODEs (J. R. Cash and I. Gladwell, eds.), Oxford Univ. Press (to appear).

13. Y. B. Suris, Some properties of methods for the numerical integration of systems of the form $\ddot{x}=f(x)$, Zh. Vychisl. Mat. i Mat. Fiz. 27 (1987), 1504-1515; English transl., U.S.S.R. Comput. Math. and Math. Phys. 27 (1987), 149-156.

14. Y. B. Suris, The canonicity of mappings generated by Runge-Kutta type methods when integrating the systems $\ddot{x}=-\partial U / \partial x$, Zh. Vychisl. Mat. i Mat. Fiz. 29 (1989), 202-211; English transl., U.S.S.R. Comput. Math. and Math. Phys. 29 (1989), 138-144.

15. H. Yoshida, Construction of high order symplectic integrators, Phys. Lett. A 150 (1990), 262-268.

Department of Computer Science, University of Illinois at Urbana-Champaign, URBANA, ILLINOIS 61801-2987

E-mail address, D. Okunbor: okunbor@cs.uiuc.edu

E-mail address, R. D. Skeel: skeel@cs.uiuc.edu 